\title{
Quantum path control in harmonic generation by temporal shaping of few-optical-cycle pulses in ionizing media
}

\author{
F. Calegari, ${ }^{1,{ }^{*}}$ M. Lucchini,${ }^{1}$ K. S. Kim,,${ }^{1,2}$ F. Ferrari, ${ }^{1}$ C. Vozzi,${ }^{1}$ S. Stagira, ${ }^{1}$ G. Sansone,${ }^{1}$ and M. Nisoli ${ }^{1}$ \\ ${ }^{1}$ Department of Physics, Politecnico di Milano, National Research Council of Italy, Institute of Photonics and Nanotechnologies (CNR-IFN), \\ Piazza L. da Vinci 32, I-20133 Milano, Italy \\ ${ }^{2}$ Department of Physics and Coherent X-ray Research Center (CXRC), Korea Advanced Institute of Science and Technology (KAIST), \\ Daejeon 305-701, Korea
}

(Received 3 May 2011; published 5 October 2011)

\begin{abstract}
Temporal reshaping of the electric field of few-optical-cycle pulses in a low-density ionizing gas has been used to achieve control of the electron trajectories in the process of high-order harmonic generation. As a result of such a quantum path control mechanism, isolated or multiple attosecond pulses have been produced, depending on the carrier-envelope phase of the driving field. In particular, complete spectral tunability of the harmonic peaks over the whole spectral range has been demonstrated. Experimental results have been interpreted using a nonadiabatic three-dimensional propagation model and a nonadiabatic stationary phase model.
\end{abstract}

DOI: 10.1103/PhysRevA.84.041802

PACS number(s): 42.65.Ky, 42.65.Re, 42.50.Hz

Extreme-ultraviolet (XUV) laser sources of isolated and trains of attosecond pulses represent very important tools for the investigation of ultrafast electron dynamics in atoms, molecules, and condensed matter, with temporal resolution approaching the atomic unit of time [1,2]. So far, high-order harmonic generation (HHG) in gases has been the workhorse technique for the production of XUV radiation in the subfemtosecond domain. In the framework of a semiclassical model [3], an electron exposed to an intense, linearly polarized electric field is emitted from the atom by tunnel ionization. The freed electron can be driven back toward its parent ion, following complex trajectories (quantum paths), governed by the electric field of the excitation pulse. Finally, electron recombination to the ground state leads to periodic emission of subfemtosecond bursts of XUV radiation. In general, two quantum paths give the most relevant contribution: the so-called short quantum paths, with an electron return time close to half an optical period, and the long quantum paths, with a return time close to one period. Control of XUV spectral and temporal characteristics requires shaping of electron trajectories on an attosecond time scale. The use of driving pulses with the stabilized carrier-envelope phase (CEP) for the confinement of the XUV generation to a single event is an example of such a quantum path control [4]. Shaped excitation pulses, synthesized by combining two of multiple wavelengths, have been also proposed and partially employed to control the quantum paths in order to enhance the energy of the XUV photons [5,6]. Moreover, phase-matching mechanisms have been used for selection and enhancement $[7,8]$ of particular quantum paths.

In this Rapid Communication we investigate, both experimentally and theoretically, a method for quantum path control on an attosecond time scale, based on the shaping of the electric field of few-optical-cycle driving pulses induced by propagation in an ionizing medium. As recently demonstrated, by using 5 -fs pulses with the above saturation intensity and controlled electric field, short temporal gates can be obtained,

\footnotetext{
*francesca.calegari@polimi.it
}

allowing the generation of nanojoule energy isolated sub-160as pulses or short trains of attosecond pulses, depending on the CEP of the driving field [9]. The physical mechanism at the basis of such a gating technique is related to the subcycle ionization dynamics in the generating medium. Here we show that such a gating technique allows one to obtain complete tunability of the XUV emission in a very broad spectral region, upon changing the CEP of the driving field. We demonstrate that the quantum path control can be interpreted as a single-atom effect related to a propagation-induced temporal distortion of the driving few-optical-cycle field. The efficient generation of tunable XUV emission over a broad spectral range is of crucial importance for various applications ranging from the seeding of free-electron lasers (FELs) to the development of unique attosecond spectroscopy methods. In the case of FEL seeding the present technique offers unique advantages: (i) full tunability between consecutive harmonic orders also at low photon energies; (ii) a nearly constant intensity of a given harmonic peak in the full tuning spectral range; and (iii) generation of XUV pulses with nanojoule energy, which is a rather demanding requirement for efficient FEL seeding [10]. Selection of single harmonics from a tunable spectrum by using time-compensated monochromators [11] allows one to perform pump andprobe time-resolved experiments in the XUV spectral region, with a few-femtoseconds temporal resolution. Generation of short trains of attosecond pulses can be important for attosecond spectral interferometry measurements [12].

The XUV radiation was generated by focusing 5-fs driving pulses at a $1-\mathrm{kHz}$ repetition rate in a $2.5-\mathrm{mm}$-long cell filled with xenon or argon at static pressure (2.5-3 Torr). The CEPstabilized driving pulses presented a CEP residual fluctuation of $\sim 120 \mathrm{mrad}(\mathrm{rms})$. The peak intensities were estimated to be $\sim 2.5 \times 10^{15} \mathrm{~W} / \mathrm{cm}^{2}$ and $3.5 \times 10^{15} \mathrm{~W} / \mathrm{cm}^{2}$ when using xenon or argon as the generating media, respectively. The gas cell was positioned just after the laser focus to select the short quantum trajectory by means of phase matching [7]. The fundamental radiation and the low-order harmonics were blocked by a 100 -nm-thick aluminum filter; a $200-\mu$ m-diam pinhole was positioned in the XUV-beam path for spatial filtering. The XUV emission was spectrally dispersed using 

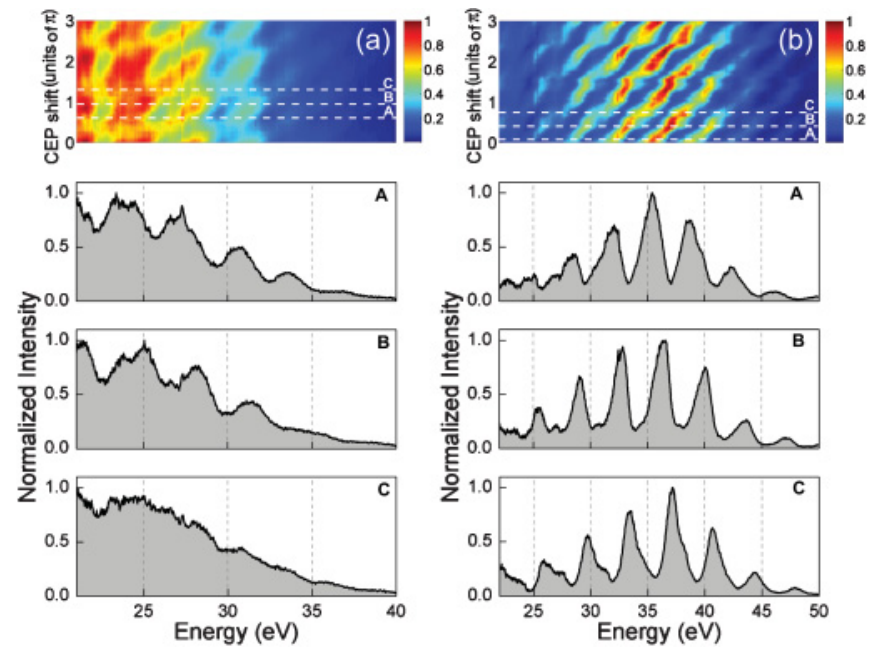

FIG. 1. (Color online) (a) Experimental XUV spectra generated in xenon (a) and in argon (b), acquired as a function of the CEP of the driving field. Bottom panels: XUV spectral profiles acquired for three different CEPs (A, B, and C).

a flat-field grazing-incidence spectrometer and detected by a microchannel plate coupled to a phosphor screen and a CCD camera [13].

Figure 1(a) shows XUV spectra generated in xenon and acquired as a function of the CEP of the driving field: The spectral shape periodically changes for a CEP shift $\Delta \phi=\pi$. For particular CEP values the XUV spectrum is continuous, thus indicating the generation of isolated attosecond pulses [9]. It is worth noting that in this case the energy of the XUV pulses was estimated to be $\sim 2 \mathrm{~nJ}$ after the aluminum filter. Upon changing the CEP value, the XUV spectrum becomes modulated as a result of the interference between two attosecond pulses. This interference gives rise to a beating structure which no longer corresponds to the odd-order harmonics of the driving field. The scan also reveals the presence of a strong shift in the XUV peak positions upon changing the CEP. We further investigated the shaping of the XUV emission as a function of the CEP by performing the same experiment in argon. Although a continuous emission can be also observed with argon as the generating medium, in this case we choose a peak intensity of the driving field that allows one to obtain a discrete emission for all the CEPs. This means that the width of the gate, which is set by the ionization profile, is large enough to select two or more attosecond pulses. Figure 1(b) shows the XUV spectra generated in argon and acquired as a function of the CEP of the driving field: A complete tunability of the XUV emission can be obtained upon changing the CEP. It is worth pointing out that complete tuning of the harmonic peaks versus CEP is not limited to the cutoff region of the XUV radiation, as already reported in previous works, where XUV tunability was restricted to the high-energy portion $(>90 \mathrm{eV})$ of the XUV spectrum $[4,14]$. Here the spectral tunability is observed over the whole XUV spectrum, which extends up to $50 \mathrm{eV}$. We have experimentally observed that CEP residual fluctuations $<200 \mathrm{mrad}$ ( $\mathrm{rms}$ ), typical for commercial CEP-stable laser systems, allows one to achieve such a tunability control.
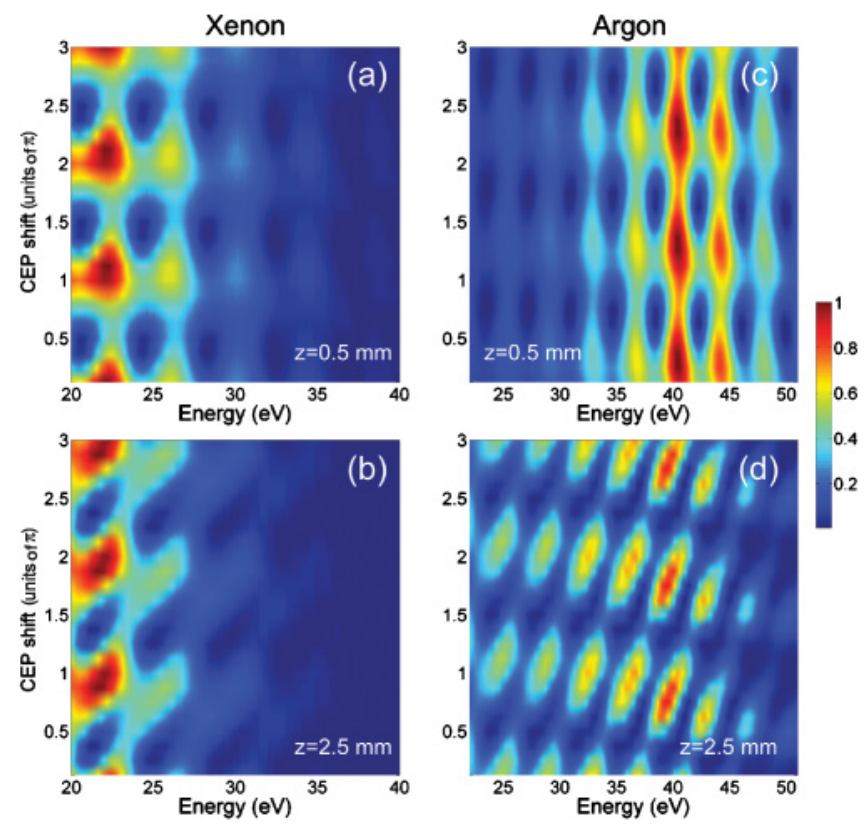

FIG. 2. (Color online) Left-hand panels: Evolution of the XUV spectra calculated using the 3D model as a function of the CEP, assuming the same parameters of the experiment, for two different propagation distances inside the gas cell filled with xenon: $0.5 \mathrm{~mm}$ (a) and $2.5 \mathrm{~mm}$ (b). Right-hand panels: Calculated evolution of the XUV spectra as a function of the CEP, assuming the same parameters of the experiment, for two different propagation distances inside the gas cell filled with argon: $0.5 \mathrm{~mm}(\mathrm{c})$ and $2.5 \mathrm{~mm}(\mathrm{~d})$.

In order to understand the physical mechanisms at the basis of the observed spectral tunability, we used a threedimensional (3D) propagation model, which takes into account both temporal plasma-induced phase modulation and spatial plasma-lensing effects on the fundamental beam [15]. The ionization rates have been calculated by using the Ammosov, Delone, and Krainov (ADK) theory [16]. Figure 2(a) shows the evolution of the XUV spectra generated in a xenon cell, calculated as a function of the CEP of the 5-fs excitation pulse, assuming the same gas pressure and laser peak intensity used in the experiment, and a gas-cell thickness of $0.5 \mathrm{~mm}$ (i.e., five times shorter than the actual thickness). In agreement with the experimental results shown in Fig. 1(a), a periodic evolution from modulated spectra to continuous spectra is clearly reproduced by the simulations but the spectral position of the harmonic peaks is almost insensitive to the CEP values, contrary to what is experimentally observed. Complete tunability of the XUV peaks is perfectly reproduced by the numerical simulation upon considering the correct gas-cell thickness, as shown in Fig. 2(b).

We performed the same calculations in the case of argon. Figures 2(c) and 2(d) show the calculated evolution of the XUV spectra as a function of the CEP of the 5-fs driving pulse, for two different gas-cell thicknesses, 0.5 and $2.5 \mathrm{~mm}$, respectively. Gas pressure and laser peak intensity were assumed to be equal to the experimental values. Also in this case, the complete tunability of the harmonic peaks can be reproduced only by considering the actual propagation in the gas cell. 

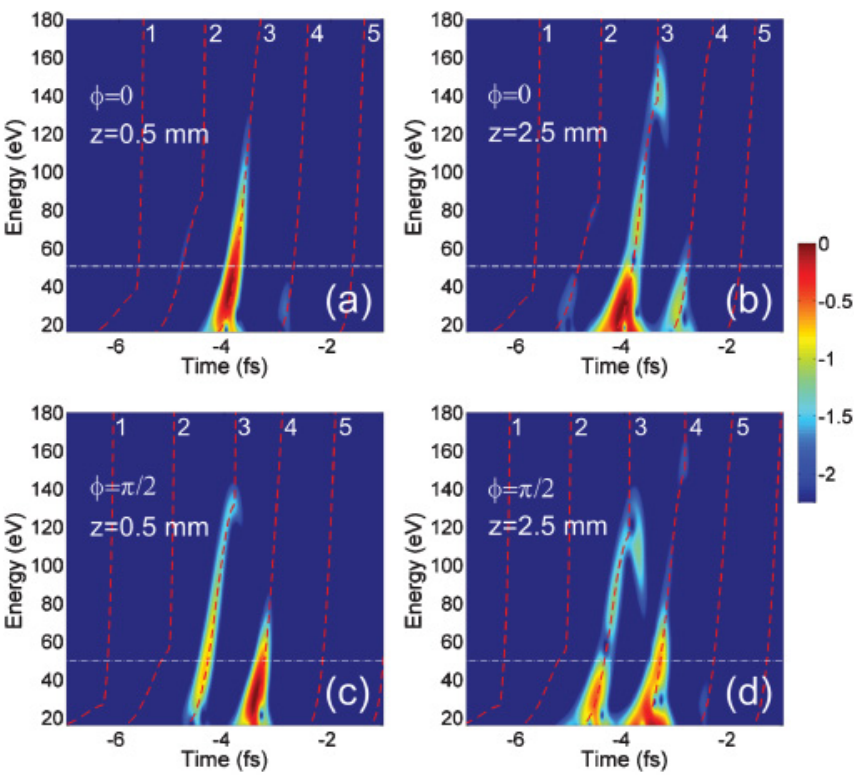

FIG. 3. (Color online) Top panels: Time-frequency spectrograms of the XUV emission from argon calculated for $\phi=0$ and after $0.5 \mathrm{~mm}$ (a) and $2.5 \mathrm{~mm}$ (b) of propagation inside the gas cell (logarithmic color map). Bottom panels: Time-frequency spectrograms of the XUV emission from argon calculated for $\phi=\pi / 2$ and after $0.5 \mathrm{~mm}$ (a) and $2.5 \mathrm{~mm}$ (b) of propagation inside the gas cell (logarithmic color map). Red dashed lines represent the emitted energies as a function of the recollision times calculated with the saddle-point analysis. Labels of the corresponding short trajectories are reported on top of the figure.

The numerical simulations show that the spatial reshaping of the driving pulse is weak, as a result of the very low pressure used in the experiment, while the plasma-induced phase modulation strongly affects the temporal shape of the electric field of the driving pulse. We will demonstrate that such a temporal distortion is responsible for the reshaping of the quantum trajectories which contribute to the HHG process.

The role of the quantum paths involved in the generation process can be analyzed by using the Gabor time-frequency analysis. This technique consists of filtering a portion of the calculated harmonic spectrum and taking its Fourier transform [17]. Figure 3 shows, in a logarithmic scale, the time-frequency spectrograms calculated in the case of argon for two CEP values $\phi=0$ and $\phi=\pi / 2$ and two gas-cell thicknesses 0.5 and $2.5 \mathrm{~mm}$. The experimental cutoff energy is shown by the white dashed lines: The overall XUV energy is almost completely contained in the photon energy range between 20 and $50 \mathrm{eV}$. In the case of the short cell, the spectrogram corresponding to $\phi=0$ [see Fig. 3(a)] exhibits a single dominant contribution: In such a case additional recollisions are strongly suppressed by the short temporal gate induced by the ionization profile. Upon considering the same gas-cell thickness, the time-frequency spectrogram corresponding to $\phi=\pi / 2$, displayed in Fig. 3(c), shows the presence of two main contributions to the XUV emission, due to the different shape of the temporal gate. Additional propagation inside the ionizing medium creates a distortion of the fundamental field, thus affecting both the shape of the temporal gate and the recollision processes. For $\phi=0$ [Fig. 3(b)] an additional contribution appears after the main one, while for $\phi=\pi / 2$ [Fig. 3(d)] two dominant contributions can be still observed, however, the temporal separation between the two is reduced by the plasma-induced chirp of the electric field. An important result of the Gabor analysis is that in all the spectrograms each energy is generated only once during a laser half cycle, corresponding to the short trajectories of the semiclassical picture. As a direct consequence we can assume that the long trajectories do not play any role in the observed spectral shift of the XUV peaks.

A more direct physical interpretation can be achieved by using nonadiabatic saddle-point simulations [18,19], which directly analyze the XUV generation process in terms of the electron trajectories. According to the results of the previous Gabor analysis, we have considered only the short quantum paths. The temporal evolution of the driving field considered in the simulations has been calculated by using the $3 \mathrm{D}$ propagation model, so that it takes into account the temporal reshaping effects induced by propagation in the ionizing medium. The calculated photon energies as a function of the recollision times for two CEP values and two propagation lengths are shown by the red dashed lines in Figs. 3(a)-3(d), showing perfect matching between the time-frequency diagrams obtained from the full 3D calculation and the results of the saddle-point simulations. We have then calculated the evolution of the XUV spectra generated in argon as a function of the CEP of the excitation pulse. In the case of an excitation field calculated at the entrance of the gas cell [hereafter called $E_{0}(t)$ ], discrete XUV spectra have been obtained, with harmonic peaks almost insensitive to the CEP of the driving field. A complete tunability of the XUV radiation, in perfect agreement with experimental results, has been obtained only considering the temporal reshaping effects induced by propagation in longer $(2.5-\mathrm{mm})$ cells [the corresponding driving electric field will be indicated as $\left.E_{2.5}(t)\right]$.

The nonadiabatic saddle-point simulations allow one to obtain a very simple physical interpretation of the observed spectral shift of the harmonic peaks. In agreement with the Gabor analysis, in our experimental condition only two quantum paths (paths 3 and 4 in Fig. 3) effectively contribute to harmonic generation. The spectral position of the harmonic peaks is determined by the phase difference $\Delta \Theta(\omega)$ between such quantum paths:

$$
\begin{aligned}
\Delta \Theta(\omega) & =\Theta_{4}(\omega)-\Theta_{3}(\omega) \\
& =\omega\left(t_{4}-t_{3}\right)-\left(S_{4}-S_{3}\right)+\left(\varphi_{4}-\varphi_{3}\right),
\end{aligned}
$$

where $t_{i}$ is the recombination time, $S_{i}$ is the semiclassical action, and $\varphi_{i}$ is a residual phase associated to the quantum path $i=3,4$ (the relative weight of the two quantum trajectories changes only the contrast of the interference pattern). We have found that the spectral shift of the harmonic peaks is almost entirely related to the first term $\omega\left(t_{4}-t_{3}\right)$ in Eq. (1), which can be also written as

$$
\omega\left(t_{4}-t_{3}\right)=\omega\left(t_{04}-t_{03}\right)+\omega\left(\tau_{4}-\tau_{3}\right)=\omega \Delta t_{0}+\omega \Delta \tau,
$$

where $t_{0 i}$ is the ionization time and $\tau_{i}$ is the time spent by the electron in the continuum, associated with the quantum path $i=3,4$. The phase term $\omega \Delta t_{0}$ is almost unaffected by 

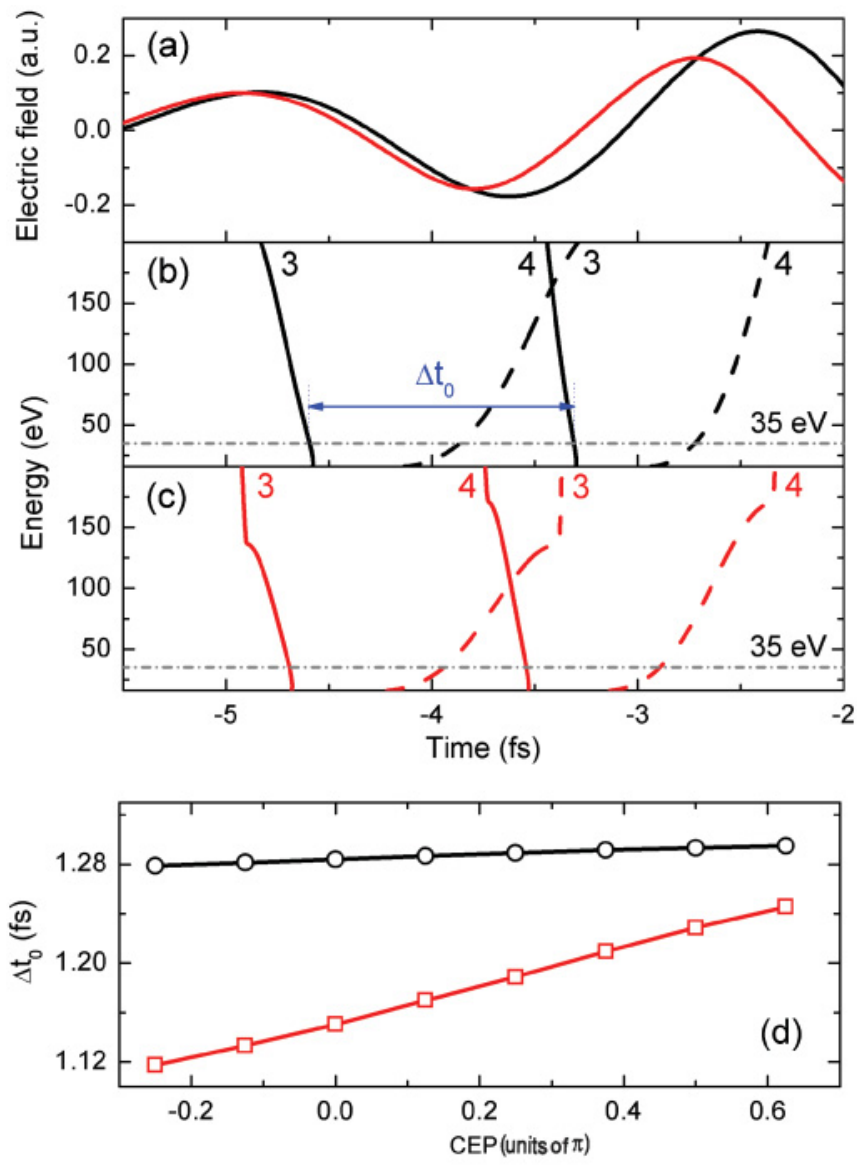

FIG. 4. (Color online) (a) Portion of the electric field calculated for $\phi=0$ at the entrance of the gas cell (black line) and after $2.5 \mathrm{~mm}$ of propagation inside the gas cell (red/gray line). Emitted photon energies as a function of the real parts of the ionization times (solid curves) and of the recombination times (dashed curves) for paths 3 and 4 calculated at the entrance of the gas cell (b) and after $2.5 \mathrm{~mm}$ of propagation inside the gas cell (c). (d) Difference of the real parts of the ionization times $\Delta t_{0}$ calculated at $35 \mathrm{eV}$ and as a function of the CEP of the driving field at the entrance of the gas cell (black circles) and after $2.5 \mathrm{~mm}$ of propagation inside the gas cell (red/gray squares).

CEP at the gas-cell entrance, while at the output it is strongly influenced by CEP. On the other hand, the phase term $\omega \Delta \tau$ shows only a small dependence on CEP in the spectral range of interest, moreover, such a contribution is almost perfectly canceled by the $\Delta S=S_{4}-S_{3}$ term, both at the entrance and at the output of the gas cell. Therefore, we can conclude that the observed harmonic tunability is mainly related to the dependence of the phase term $\omega \Delta t_{0}$ on CEP, produced by the temporal distortion of the electric field induced by the propagation inside the gas cell, as shown in Fig. 4(a). The effects of such a temporal distortion on the quantum trajectories can be clearly observed in Figs. 4(b) and 4(c), where the real parts of the ionization times (solid curves) and recombination times (dashed curves) for paths 3 and 4 are reported assuming $E(t)=E_{0}(t)$ and $E(t)=E_{2.5}(t)$, respectively. In order to give a more direct picture of the physical mechanism, we have calculated the evolution of $\Delta t_{0}$ at the fixed energy of $35 \mathrm{eV}$ as a function of the CEP of the driving field: The results are shown in Fig. 4(d). At the entrance of the gas cell $\Delta t_{0}$ is almost insensitive to CEP, while at the output of the cell $\Delta t_{0}$ linearly increases with CEP: Such an evolution is connected to the plasma-induced chirp of the electric field. Thus, the tunability of the XUV emission, observed over the whole spectral range, can be completely assigned to the reshaping of the quantum trajectories as a result of the propagation in the ionizing medium.

In conclusion, in this Rapid Communication we demonstrated the possibility to completely control the XUV emission process upon changing the CEP of an intense few-cycle driving field. The reshaping of the electron quantum trajectories induced by nonadiabiatic propagation in an ionizing medium allows one to obtain complete tunability of the XUV emission over the whole spectral range. It is worth noting that as only two trajectories contribute to the XUV emission, this spectral shaping in the frequency domain corresponds in the time domain to the generation of two attosecond pulses, whose relative phase can be controlled by adjusting the CEP of the driving pulse. Such a behavior can be a promising tool for attosecond transient-absorption spectroscopy experiments [12].

This research was supported by the European Research Council under the European Community's Seventh Framework Programme (FP7/2007-2013)/ERC Grant Agreement No. 227355 ELYCHE, the Italian Ministry of Research (FIRBIDEAS RBID08CRXK), the European Union under Contract No. 228334 JRA-ALADIN (Laserlab Europe II), and the MC-RTN ATTOFEL (FP7-238362). The authors gratefully acknowledge V. Tosa and K. Kovács for fruitful discussions.
[1] F. Krausz and M. Ivanov, Rev. Mod. Phys. 81, 163 (2009).

[2] M. Nisoli and G. Sansone, Prog. Quantum Electron. 33, 17 (2009).

[3] P. B. Corkum, Phys. Rev. Lett. 71, 1994 (1993).

[4] A. Baltuška, Th. Udem, M. Uiberacker, M. Hentschel, E. Goulielmakis, Ch. Gohle, R. Holzwarth, V. S. Yakovlev, A. Scrinzi, T. W. Hänsch, and F. Krausz, Nature (London) 421, 611 (2003).

[5] F. Calegari, C. Vozzi, M. Negro, G. Sansone, F. Frassetto, L. Poletto, P. Villoresi, M. Nisoli, S. De Silvestri, and S. Stagira, Opt. Lett. 34, 3125 (2009).
[6] L. E. Chipperfield, J. S. Robinson, J. W. G. Tisch, and J. P. Marangos, Phys. Rev. Lett. 102, 063003 (2009).

[7] Y. Mairesse, A. de Bohan, L. J. Frasinski, H. Merdji, L. C. Dinu, P. Monchicourt, P. Breger, M. Kovaĉev, R. Taieb, B. Carré, H. G. Muller, P. Agostini, and P. Salières, Science 302, 1540 (2003).

[8] T. Popmintchev, M.-C. Chen, P. Arpin, M. M. Murnane, and H. C. Kapteyn, Nat. Photon. 4, 822 (2010).

[9] F. Ferrari, F. Calegari, M. Lucchini, C. Vozzi, S. Stagira, G. Sansone, and M. Nisoli, Nat. Photon. 4, 875 (2010). 
[10] B. W. J. McNeil, J. A. Clarke, D. J. Dunning, G. J. Hirst, H. L. Owen, N. R. Thompson, B. Sheehy, and P. H. Williams, New J. Phys. 9, 82 (2007).

[11] L. Poletto, P. Villoresi, E. Benedetti, F. Ferrari, S. Stagira, G. Sansone, and M. Nisoli, Opt. Lett. 29, 86 (2004).

[12] T. Pfeifer, M. J. Abel, P. M. Nagel, A. Jullien, Z. Loh, M. J. Bell, D. M. Neumark, and S. R. Leone, Chem. Phys. Lett. 463, 11 (2008).

[13] L. Poletto, G. Tondello, and P. Villoresi, Rev. Sci. Instrum. 72, 2868 (2001).

[14] M. Nisoli, G. Sansone, S. Stagira, S. De Silvestri, C. Vozzi, M. Pascolini, L. Poletto, P. Villoresi, and G. Tondello, Phys. Rev. Lett. 91, 213905 (2003).
[15] E. Priori, G. Cerullo, M. Nisoli, S. Stagira, S. De Silvestri, P. Villoresi, L. Poletto, P. Ceccherini, C. Altucci, R. Bruzzese, and C. de Lisio, Phys. Rev. A 61, 063801 (2000).

[16] M. V. Ammosov, N. B. Delone, and V. P. Krainov, Zh. Eksp. Teor. Fiz. 91, 2008 (1986) [Sov. Phys. JETP 64, 1191 (1986)].

[17] V. S. Yakovlev and A. Scrinzi, Phys. Rev. Lett. 91, 153901 (2003).

[18] G. Sansone, C. Vozzi, S. Stagira, and M. Nisoli, Phys. Rev. A 70, 013411 (2004).

[19] K. Kovács and V. Tosa, J. Mod. Opt. 57, 977 (2010). 